\title{
Tras las huellas de Jorge Juan: una mirada geográfica sobre Francisco José de Caldas
}

Foolowing in Jorge Juan's traces: a geographical point of view about Francisco José de Caldas

\author{
Luz Marina Duque Martínez \\ Departamento de Filosofia/Universidad del Valle
}

\section{Resumen}

Francisco José de Caldas hizo parte del grupo de criollos ilustrados que desempeñaron un papel destacado en el período anterior a la independencia de Nueva Granada. El neogranadino tenia gran afición por las ciencias, especialmente por la astronomia. El momento histórico en el cual vivió Francisco José de Caldas hizo que su gusto por las ciencias pasara del mero gusto por el saber a la conciencia de lo que ese saber significaba. Los ilustrados de la península ibérica habían acuñado el concepto "ciencia útil" para promover y estimular el desarrollo de la educación y del saber en los territorios españoles peninsulares y de ultramar. Don Francisco José hizo suyo el concepto de "ciencias útiles", tan caro a los ideales ilustrados españoles. En nuestro concepto, fue esa circunstancia la que lo hizo transitar de la astronomía observacional y teórica a la astronomía práctica, fundamentalmente, a la geodesia y a la cartografia. Se trata de un científico criollo educado en el paradigma de la Física de Newton y de la Astronomía consecuente con ella; un científico, que en su etapa de estudiante, recibió conocimientos de Geometría esférica, que le fueron muy útiles para realizar los cálculos que las determinaciones astronómicas le exigirian.

\section{Abstract}

Francisco Jose de Caldas was a member of the group of learned nativeborn persons who played an outstanding role in the period previous to the independence of New Granada. The neogranadian was very fond of sciences, specially, the astronomy. The historical moment in which Fran- 
cisco Jose de Caldas lived made his liking of sciences passed from the mere predilection for knowledge to the conscience of what this knowledge meant. The erudites of the Iberian peninsula had coined the concept "useful science" to promote and to stimulate the development of education and knowledge in the Spanish peninsular and overseas territories. Don Francisco Jose appropriated the concept of "useful sciences", so expensive for the erudite Spanish ideals. In our concept, this circumstance made him leave behind the observational and theoretical astronomy, and turn his thoughts to the practical astronomy, fundamentally, the geodesy and the cartography. He is a native-born scientist educated in the Newton Physics paradigm and the Astronomy consistent with it; a scientist, who in his student's period, received knowledge of spherical Geometry, that was very useful for him to do the calculations that the astronomic determinations would demand him.

Key words: Botanic expedition, Astronomy, cartography, Geography, astronomic observatory, planet system.

Sabio y Meritorio son adjetivos con los que ha sido calificado Francisco José de Caldas. Como "el sabio Caldas" suele denominársele en nuestra tradición colombiana, casi siempre desconociendo los motivos que ameritarian esta calificación y, por tanto, corriendo el riesgo de que se convierta en una denominación vacía. En 1802, en una carta a Mutis, el mismo Caldas decía "No soy sabio, este es mi primer conocimiento"; y añadía que cuando se lo calificaba de esa manera se le negaba la posibilidad de continuar con su instrucción que él consideraba insuficiente. Como Meritorio lo calificó Mutis en carta que le dirigió a Cavanilles, Director del Jardín Botánico de Madrid, en julio del mismo año de 1802, cuando le comunicó que lo había "agregado a mi Expedición en calidad de Meritorio".

Francisco José de Caldas nació en Popayán en Octubre de 1768. Era hijo de un español, Josef de Caldas y una criolla, Vicenta Thenorio Arboleda. Realizó sus primeros estudios en el Colegio Seminario de San Francisco de Asís, único colegio existente en Popayán. Allá llegó José Félix de Restrepo en 1782 y cumplió una meritoria labor tanto en la renovación de los métodos de enseñanza, como de los contenidos de las mismas. Corrian los tiempos del Reinado de los Borbones en España y 
de los virreyes ilustrados en Nueva Granada. Conviene ahora que digamos algunas palabras acerca de otro de los "mentores" de Caldas.

José Celestino Mutis, naturalista español, había llegado a Nueva Granada como médico personal del Virrey Messía de la Cerda, en 1760. Según su biógrafo, Mutis decidió abandonar una prometedora carrera en Europa porque le seducía más la exploración y conocimiento de la naturaleza americana. La Expedición Botánica del Nuevo Reino de Granada no tuvo carácter itinerante, sino que se convirtió en la institución científica más importante del período colonial. Mutis le presentó un proyecto al Arzobispo Virrey Antonio Caballero y Góngora, quien la fundó provisionalmente en 1782 mientras esperaba recibir la sanción del Rey, que llegó al año siguiente; la Expedición estuvo vigente hasta 1811, un poco después del primer movimiento de independencia. Caldas no estuvo entre los primeros colaboradores de la Expedición Botánica, de quienes cabe destacar a Francisco Antonio Zea, Jorge Tadeo Lozano, José Joaquín Camacho, Miguel Pombo, José Maria Carbonell y Sinforoso Mutis.

Caldas estuvo en Santafé de Bogotá entre 1788 y 1792 haciendo su Bachillerato en Derecho en el Colegio del Rosario. Todo parece indicar que en esa época no entró en contacto con Mutis. Al menos así lo sostiene argumentadamente Santiago Díaz Piedrahita. ¿Cómo llegó Caldas a la Expedición Botánica? Todo parece indicar que fue por el conocimiento que tuvo Mutis de los trabajos científicos de Caldas a través de Santiago Arroyo, uno de los amigos más cercanos del payanés, que residía en Santafé. Mutis decidió agregarlo a la Expedición en Marzo de 1802, y para ese efecto le dirigió una comunicación a Quito, donde se encontraba. Desde el momento en que Mutis vinculó a Caldas a la Expedición, le asignó la dirección del Observatorio Astronómico, del que debería encargarse tan pronto llegara a Santafé, tras cumplir tareas botánicas en Quito y sus alrededores.

\section{Caldas, criollo ilustrado}

Sus concepciones filosóficas y metodológicas acerca de cómo es la naturaleza y cómo puede ser conocida, cuáles son los límites de la autoridad de los autores antiguos, cómo deben relacionarse los campos de la razón y la fe, nos lo muestran como un ilustrado español. Como ilustrado español, también acogió la concepción de "ciencias útiles" adoptada en la península en aquellos tiempos. 
Caldas consideraba que la naturaleza, como se solía decir entonces, poseía un orden, que no era accidental sino la expresión de una inteligencia ordenadora. Pensaba que el conocimiento no provenía de la experiencia inmediata, sino de la indagación y del esfuerzo de comprender las relaciones presentes en los fenómenos de la naturaleza, que no eran evidentes. A esa comprensión fue a la que los ilustrados llamaron "arrancarle los secretos a la naturaleza", porque se lograba a través de la formulación de hipótesis y de observaciones atentas y no como resultado de un golpe de intuición. Como devoto cristiano, Caldas entendia su fe como un asunto diferente de la investigación acerca de la naturaleza. Fue un observador muy cuidadoso, casi al límite de la meticulosidad, y se enorgullecía de ello, porque consideraba que ese cuidado garantizaba mayor precisión en sus observaciones. Más de una vez se sintió con derecho a criticar a otros científicos que no guardaban las mismas precauciones que él. Ni siquiera Mutis escapó a sus críticas.

En Observaciones Astronómicas de Jorge Juan, encontramos ese mismo espíritu de precisión. Pero, los desarrollos instrumentales y teóricos en la época de la Expedición Geodésica al Perú, hacían que el cuidado por la precisión fuese más una decisión metodológica que una tarea que efectivamente pudiera llevarse a cabo. En varios escritos de Caldas encontramos observaciones acerca de las imprecisiones en la determinación de la longitud o la latitud de un lugar, por parte de los expedicionarios o alguna otra imprecisión en la determinación de la altura de otro lugar cualquiera. Pareciera que Caldas se hubiera impuesto como tarea verificar las mediciones realizadas por los expedicionarios venidos de Europa.

\section{¿Cómo se convirtió Francisco José de Caldas en astrónomo?}

La aparición de los Principios Matemáticos de Filosofia Natural de Newton en 1687, había marcado una nueva dirección en el desarrollo de la Física y de la Astronomía. Newton estableció los principios básicos de la mecánica, hoy conocida como clásica; estableció los principios básicos de la dinámica de fluidos; dedujo las leyes del movimiento planetario de la Ley de Gravitación; explicó el movimiento de la Luna; explicó las mareas; explicó las órbitas de los cometas; calculó las masas de la Tierra, del Sol y de los planetas; explicó la forma achatada de la Tierra y señaló su relación con la precesión de los equinoccios. 
Los horizontes de investigación astronómica abiertos por Newton alentaron los desarrollos astronómicos durante un largo período. Consistian, fundamentalmente, en el desarrollo de la mecánica celeste, que permitiria hacer predicciones, más precisas que nunca, acerca del movimiento y posición de los astros. Durante la segunda mitad del siglo XVIII se pusieron en juego todos los recursos teóricos y prácticos para consolidar la astronomía como ciencia. Se trató de un inmenso programa de investigación que permitió definir el perfil de la astronomía en el primer cuarto del siglo XIX; a la definición de ese perfil contribuyó la aparición del Traité de Méchanique Céleste de Laplace, publicado entre 1799 y 1825; Laplace reunió en un sólo cuerpo teórico, los trabajos dispersos de Newton, Halley, Clairaut, D'Alembert, Euler y los suyos propio. De esta manera, recogió el conocimiento de su época sobre el movimiento de los cuerpos del Sistema Solar.

El aspecto práctico del programa de investigación astronómica derivado de los trabajos de Newton fue promovido por la expansión económica y comercial de Europa, tuvo carácter náutico, cartográfico e hidrográfico. Ambos aspectos del programa estuvieron relacionados entre sí en muchos niveles. El desarrollo de la mecánica celeste permitió alcanzar mayores niveles de precisión en la predicción de los fenómenos celestes y con ello, contribuyó a hacer cada vez más segura la navegación. Los gobiernos requerían personal preparado y eso los llevó a apoyar la fundación de observatorios astronómicos; así mismo, la necesidad de contar con buenos instrumentos para las tareas cartográficas e hidrográficas estimuló la industria de la construcción de instrumentos científicos, conduciéndola a la fabricación de mejores y más precisas herramientas cientificas.

Ocupémonos ahora a nuestro científico criollo. Si tuviéramos que responder a la pregunta dónde aprendió Caldas sus primeras nociones de astronomía y sus primeros conocimientos de geometría y especialmente, las propiedades de la esfera, tendríamos que decir que fue en el Seminario de San Francisco de Asís, en Popayán, donde recibió lecciones de José Félix de Restrepo, el "catedrático ilustrado" que Caldas le mencionó a Mutis en la primera carta que le escribió, y quien le enseñó Aritmética, Geometría, Trigonometría, Álgebra, Física Experimental y Astronomía. 
Lecciones de Física Experimental, el texto con el que Restrepo enseñaba Física, no es un libro de Física solamente, es un Compendio de Ciencias Naturales, abarca la Física propiamente dicha, la Geografia, la Geodesia, la Astronomía, la Anatomía y la Biología. A través de ese texto Restrepo trató de ilustrar a los estudiantes acerca de las novedades en la investigación científica y de una de las más importantes expediciones científicas que ocurrió en nuestro territorio: La Expedición Geodésica al Perí, patrocinada por la Academia de Ciencias de París, para definir la verdadera figura de la Tierra. Esta expedición científica a tierras americanas se convirtió en un parangón para Francisco José de Caldas. Deseó ardientemente recorrer los lugares que recorrieron los expedicionarios y lo hizo; se propuso verificar la exactitud de las observaciones y de los registros, herencia de aquella expedición y sobre todo, el informe de Jorge Juan, conocido como Observaciones Astronómicas, se constituyó en guia para la realización de sus propias correrias científicas.

Terminada la primera etapa de estudios, fue enviado por su padre al Colegio del Rosario para que se hiciera Jurisconsulto; allá permaneció desde 1788 hasta 1792, cuando obtuvo el título de Bachiller en Derecho. En adelante, su formación fue autodidacta. Su correspondencia nos da un fiel testimonio de su incansable búsqueda de libros e instrumentos. Tanto en sus memorias científicas como en su correspondencia, Caldas fue registrando los autores y títulos de los libros que consultaba y que le servían de referencia. En cuanto a fuentes bibliográficas para el estudio de los cielos se refiere, después de 1796 cuando lo consultó por primera vez en Santafé, Caldas volvió a tener acceso a la Astronomía de Lalande, en Quito, cuando Humboldt le prestó el libro para que lo estudiara y pudiera copiar los nombres de 500 estrellas. Sabemos que leyó y estudió Observaciones Astronómicas de Jorge Juan. Encontramos citado a Delisle, Mémoire pour servir á l'Histoire et aux progres de l'Astronomie; a Flamsteed, Historia Celestial Británica, a Lacaille, Astronomie Fundamenta; a Maskelyne, Almanaque Náutico; Almanaque Náutico de Cádiz y El Conocimiento de los tiempos del Observatorio de París, de donde tomó la Tabla de diferencias de meridianos en tiempo y grados. También sabemos que leyó La figure de la Terre de Bouguer, a partir de la cual aprendió a calcular altitudes mediante la lectura del barómetro y Mens des trois degres e Introduction Historique de La Condamine.

Lo anterior nos indica que, con el paso del tiempo, Caldas se puso al 
dia en los conocimientos astronómicos de su época. Probablemente no tuviera suficiente información y medios para producir conocimientos nuevos en este campo, pero sí para aplicar los conocimientos existentes, especialmente en el terreno de la astronomía "práctica", cuyas principales ocupaciones en el siglo XVIII fueron: medir la longitud de los reinos, localizar en longitud y latitud las ciudades importantes, delimitar territorios; tareas todas a las que dedicó sus esfuerzos don Francisco José. ¿Se diferencia mucho el trabajo que hizo del que hacían los astrónomos de la época? ¿Acaso no se dedicaban también a observar eclipses de sol y de luna y de los satélites de Júpiter? ¿Acaso no les preocupaba la refracción y las mediciones barométricas?

Una buena hipótesis para analizar la dimensión del trabajo científico del autor que venimos estudiando, es pensar que la adopción del carácter práctico de la astronomía, por parte de Caldas fue como una especie de destino: era la única manera de ser astrónomo en América en la época en la cual vivió. Parece que él mismo fue consciente de su situación. Veamos lo que le escribió a Santiago Arroyo: "Mi don Santiago: si tuviéramos los instrumentos más necesarios, hariamos mucho; trabajamos en un terreno virgen, en que podemos ser originales. ¡Si Mutis se franqueara, si nos ayudara con uno u otro instrumento que sobran en su casa, si nos diera algunos libros necesarios!"

Caldas fue un científico criollo educado en el paradigma de la Física de Newton y de la Astronomía consecuente con ella; un científico, que en su etapa de estudiante, recibió conocimientos de Geometría esférica, que le fueron muy útiles para realizar los cálculos que las determinaciones astronómicas le exigirian.

\section{Predilección por la Astronomía}

Fueron muchos los escritos en los cuales Caldas habló de su predilección por la astronomía, de cómo la contemplación de los cielos lo hacia sentirse más cerca de su hacedor; fueron muchas también las cartas en las que dio cuenta de sus observaciones astronómicos e instó a sus amigos a seguir el ejemplo.

Por su correspondencia nos enteramos que desde el año de 1795 cuando decidió hacerse mercader con el propósito de ayudar a su familia, yendo de un sitio para otro por caminos de herradura, observaba esos caminos más con ojos de científico que de mercader; cuando bajo esta 
última condición visitó Santafé en 1796 aprovechó este viaje para proveerse de libros e instrumentos, tal como se lo contó a Mutis en la primera carta que le escribió.

En carta a su amigo Santiago Arroyo, escrita el 5 de Junio de 1797, don Francisco José le contaba que había comenzado a realizar observaciones de latitud en Popayán, su ciudad natal. Año y medio después, el 5 de Diciembre de 1798, en otra carta al mismo amigo, le contaba que había estado observando el Sol y algunas estrellas con el fin de calcular la latitud de Tímaná, le informaba que ya disponía del Almanaque Náutico, que había adaptado un telescopio para observar los eclipses de los satélites de Júpiter, lo que le permitiría no sólo hacer determinaciones de latitud sino también de longitud. No obstante, le pedía a su camarada que le consiguiera un telescopio del que había tenido noticias y se encontraba en Cali.

La correspondencia del payanés nos brinda mucha información acerca de sus gustos y preferencias intelectuales, así como de sus proyectos científicos. En la primera carta que le escribió a don José Celestino Mutis, el 5 de agosto de 1801 , le hablaba de la educación que había recibido y de sus intereses científicos, en particular de su preferencia por la astronomía y de su reconocimiento de las dificultades que entrañaba ser astrónomo en América, debido a la escasez de libros e instrumentos; según el mismo Caldas, la conciencia de estas limitaciones lo había decidido a dirigir sus esfuerzos hacia la botánica. Dejemos que sea don Francisco José quien hable:

"Nada tocaba más vivamente mi gusto que la astronomía; su relación con la navegación, con la geografia, con la cronología, lo brillante y magnifico del espectáculo me decidieron por ella. ¿Pero qué podia hacer en un país en que se ignoran hasta los nombres de cuarto de círculo, telescopio y péndola? Cuatro libros que una feliz casualidad arrojó a esta ciudad, me daban nociones de esta ciencia y de sus instrumentos; mis deseos, mi furor por la astronomía me sugerian recursos. Un pequeño gnomon que hice construir me entretenía; tiraba meridianos, observaba alturas del Sol, fijaba latitud, calculaba azimudes, y emprendi conocer la amplitud de la eclíptica por la observación de los solsticios; con sólo este instrumento estaban para mí como aniquiladas las estrellas y los planetas, y no podía dar un paso más en la ciencia que hacia mis delicias. (...) Volvi el año de 1796 a Santafé con miras de mercader: aqui 
vi por la primera vez y de paso la astronomía de Lalande y los elementos del Abate Besout para los guardias marinas de Francia. Estos dos libros, al tiempo que me instruian, me manifestaban que era imposible ser astrónomo en América. Copié del último las tablas del Sol para calcular sus declinaciones y hacerlas servir en mis observaciones de latitud; compré una brújula, un barómetro de mar, dos termómetros y un octante de reflexión".

Resulta interesante que don Francisco José en 1796 conociera la existencia de la Astronomia de Lalande, publicada por primera vez en París en 1764; esta obra abarcaba todos los conocimientos astronómicos y fisicos de la época, necesarios para que astrónomos aficionados y profesionales realizaran su tarea.

Instalado en el Observatorio Astronómico, como Director del mismo, en 1808 le escribió a Jerónimo Torres Tenorio, hermano de Camilo Torres, unos consejos, que refrendaba con la autoridad de "16 años dedicados a la astronomía". Caldas se regocijaba de que Jerónimo ya tuviera un sextante, un octante y una aguja azimutal y que estuviera haciendo trámites para conseguir un telescopio acromático; se alegraba porque le daba esperanzas que, por fin, tendría un corresponsal. Le indicaba el tipo de observaciones que deberá realizar cuando tuviera el telescopio: ocultaciones de estrellas por la Luna, eclipses de Sol, eclipses de los satélites de Júpiter. Y le aconsejaba que adquiriera también un buen reloj porque el tiempo era muy importante para la astronomía.

En el primer Informe al Virrey Amar y Borbón que Caldas escribió en 1809, después de la muerte de Mutis, le manifestó la misma idea, con la que se había presentado ante Mutis por primera vez: "Nacido con una inclinación irresistible por las Matemáticas, y en especial por la Geografia y por la Astronomía, me dediqué desde mis primeros años a ese estudio. La falta absoluta de libros, de instrumentos y de maestros, detuvo mis primeros pasos".

Habian pasado once años desde cuando le escribió a Santiago Arroyo rogándole le consiguiera un buen telescopio, siete, de la primera carta a Mutis, uno de la carta a Jerónimo Torres, cuando en 1809, obligado a presentar informes de sus trabajos científicos ante la máxima autoridad del Virreinato, Caldas nuevamente habló de su predilección por los estudios astronómicos.

Otros siete años después, en la última carta que escribió, dirigida a 
Pascual Enrile y Alcedo, en octubre de 1816, se presentaba asi: "Un astrónomo desgraciado se dirige directamente a Vuestra Excelencia sin otro mérito que el saber que Vuestra Excelencia profesa las ciencias exactas y conoce su importancia y su mérito. (...) Toda mi vida la he consumido, señor, en cultivar la astronomía aplicada a la geografia y la navegación, a la física y a la historia natural; comencé a persuadirme que había acertado en esta carrera cuando vi el aprecio que hicieron de mis trabajos el señor don José Celestino Mutis y el Barón de Humboldt, y comenzaron a dispensarme su protección y favores."

Caldas quería congraciarse con Enrile, a fin de que intercediera por su vida; se definió como un astrónomo y apeló a lo que consideraba tenian en común: el interés por las ciencias; Caldas sabía que, en tanto marino, Enrile había participado en las operaciones geodésicas de medidas en Cataluña. Sin embargo, no nos ocuparemos aquí ni de las intenciones ni del significado de la última carta que escribió el neogranadino; lo único que queremos destacar es que, aún enfrentado a la muerte, seguía viéndose a sí mismo como un astrónomo, que habia dedicado sus esfuerzos a la parte práctica de la astronomía.

Podriamos decir que el científico payanés nunca abandonó sus intereses astronómicos, aunque sí los reorientó hacia la geografia y la cartografia. Eso, al menos, es lo que nos hacen pensar sus palabras: "Toda mi vida la he consumido (.. ) en cultivar la astronomía aplicada a la geografia y la navegación, a la física y a la historia natural".

\section{Las Observaciones Astronómicas, de Jorge Juan: Un modelo a seguir}

Don Francisco José leyó Observaciones Astronómicas, de Jorge Juan y en su correría por los territorios de lo que hoy es Ecuador, se esforzó en comparar las observaciones que él realizaba con aquéllas que habian realizado los expedicionarios al Perú. Cuando analizamos las precauciones que tomaba el científico criollo, al hacer sus determinaciones de latitud y longitud no podemos menos que pensar que el cuidado en las mediciones barométricas, la atención a la refracción, la importancia de determinar la oblicuidad de la eclíptica y otras más, fueron aprendidas en el texto de Juan. Una misión científica tan importante como fue aquella expedición, realizada, además, en nuestras propias tierras, despertó el interés y el deseo de emulación por parte de los científicos locales. En la ya mencionada primera carta a Mutis, le contaba cómo el hecho de tener 
que ir a Quito a resolver unos pleitos de su familia, hizo renacer nuevamente en él su pasión por la astronomía. Y añadia: "Este pais visitado por los héroes de esta ciencia, que han determinado la elevación y posición de estos lugares y que han dejado los monumentos más preciosos. Estos me arrastraban con más violencia que el oro y todas las riquezas: este país es un libro abierto en que puede estudiar un aficionado a las matemáticas."

En 1801 cuando lo agregó a la Expedición Botánica, Mutis le asignó algunas tareas de astronomía práctica. Muy probablemente el celo que Caldas puso en cumplir esta tarea no se debiera solo al respeto que le inspiraba Mutis, sino a su propio deseo de constatar, a través de sus propios cálculos, las mediciones que habian realizado los expedicionarios franceses y españoles en Perú. Indudablemente la ciudad de Quito llamaba poderosamente la atención de Caldas. En Memoria sobre el plan de un viaje proyectado de Quito a la América Septentrional, presentada al célebre Director de la Expedición Botánica de la Nueva Granada, Don José Celestino Mutis, por F.J. Caldas, en 1801, decía:

"Quito, que sin contradicción es la más bella porción de la América Meridional, merece ser visitada con atención particular. Si su posición la distingue de todos los pueblos, sus producciones naturales, sus volcanes, la espantosa cordillera de que son parte, sus antiguos habitantes, los presentes, y el haber servido de teatro a un viaje célebre, llaman a los sabios de todas las partes de la tierra. Sería una desidia reprensible partir de esta Provincia sin conocerla. ¡Qué objetos presentes tan dignos de un filósofo!"

Caldas hizo muchas alusiones y reflexiones a propósito de la Expedición Geodésica a Perú; en una nota de pié de página en Del influjo del clima sobre los seres organizados (1808), hizo una breve y acertada exposición acerca de la Expedición Geodésica, que nos revela a un Caldas conocedor de la teoría newtoniana y enterado de las discusiones físicas y astronómicas más importantes de su época.

\section{Trabajos Astronómicos}

Tenemos, pues, que mientras Mutis prepara las condiciones y el futuro lugar de trabajo de Caldas, éste se dedica a hacer observaciones de fenómenos astronómicos y metereológicos, en Popayán, su ciudad natal, en la hacienda de Paispamba, de propiedad de su familia y en las 
correrías que realizó desde Popayán hasta Loja, en el sur de Ecuador.

Ya en una fecha temprana, Diciembre de 1795, en carta que le escribió a su amigo Santiago Arroyo le hablaba del entusiasmo por el conocimiento que le despertaba el contacto con la naturaleza. Aunque no había sido el interés por el conocimiento lo que lo había llevado a recorrer por los caminos de la Nueva Granada, al transitar por ellos y tener la oportunidad de ver plantas, ríos, animales, colinas y todo lo que encontraba a su paso, se dispuso a realizar y registrar observaciones por su propia cuenta.

En Junio de 1797, en carta al mismo amigo, le comunicaba sus primeros cálculos de latitud:

"Antes de mi partida de esta ciudad [Popayán] he comenzado algunas observaciones de latitud, después de haber tirado con el mayor escrúpulo una meridiana. En ella observé la declinación de la brújula, punto de la mayor importancia para levantar la carta de esta ciudad y para la que proyecto desde Popayán hasta Fortalecillas, en que acaba la que incluyo. Hallé que declinaba $8^{\circ} 10^{\prime}$ nordeste. El 23 de mayo comencé una observación de latitud, y el resultado me llenó de satisfacción al verla casi coincidir con la que hizo en años pasados $\mathrm{M}$. Buiver[sic]; éste halló $2^{\circ} 27^{\prime}$ y yo $2^{\circ} 30^{\prime}$ y algunos segundos."

En sus Observaciones Astronómicas don Jorge Juan escribió: "Los eclipses de Luna son también muy propios para determinar la Longitud de los lugares, haciendo igual uso de ellos, que de las inmersiones de los satélites; por cuyo motivo tuvimos gran cuidado, en observar todos los que pudimos en el discurso del viaje."

En Diciembre de 1797, Francisco José anunció que iba a observar un eclipse de Luna que le permitiria corregir la posición de muchos lugares de las cartas que había levantado de Bogotá a Neiva, de Popayán a La Plata y de la Jurisdicción del Cabildo de Timaná. En Enero de 1798 relató que observó el eclipse y determinó un punto en longitud, por medio de la inmersión y emersión de un gran número de lugares y manchas de la Luna. Solicitó un alfabeto griego..., "Ya sabe usted que los astrónomos señalan las estrellas con caracteres griegos, desde su inventor Bayeno: yo conozco la figura de estos caracteres, y no se el nombre de ellos para poderlos enunciar. Espero me remita usted un alfabeto griego, bien formado, trayendo cada letra su nombre al lado y su correspondiente en nuestro alfabeto." Lo encontramos nuevamente en Marzo de 1801 preparándose para observar otro eclipse de Luna, desde Popayán. Aspi- 
raba establecer, sin lugar a dudas, la posición de su ciudad natal. Pero las nubes le jugaron una mala pasada!

Desde Diciembre de 1798 , podría decirse que había comenzado a perfilarse su programa de investigación científica: se disponia a hacer las observaciones necesarias para levantar una carta completa del Virreinato, para lo cual ya tenía en sus manos el Almanaque Náutico. Fué en esta carta donde enunció la relación entre la astronomía y la geografia. Leamos:

"Yo me aflijo cuando veo tan poca astronomía en toda la extensión de la Nueva Granada, y que no hay uno a quien se le pueda encargar observe una latitud. Todo ha de salir de los libros, y ilos libros no los han formado el descuido y la ignorancia de esta materia? Por un ejemplo podrá usted juzgar. Piedrahita [Lucas Fernández de Piedrahita], en su historia de este Reino, coloca a la villa de Timaná a los $2^{\circ} 30^{\prime}$ de latitud norte; y yo, por cuatro observaciones acordes, dos con el sol, y por diferentes métodos, y dos con las estrellas, le hallé de $1^{\circ} 58^{\prime}$, es decir, 72' más corta. Después de esto, ¿qué confianza podré tener de unas determinaciones tan absurdas? ¿Y si no echamos manos de esos datos, de qué nos serviremos para formar la carta proyectada? Es preciso observar, es necesario levantar al cielo los ojos para poder ver la tierra, según expresión de Juan Andrés."

Asi pues, en 1798, Caldas se propuso observar diez y seis eclipses de los satélites de Júpiter, que habían sido calculados para el Observatorio de Cádiz y que redujo a las coordenadas de Popayán. En esta época ya disponía de un telescopio, al que él mismo le había hecho algunas adaptaciones. No obstante, a medida que se fue afinando como observador, se hizo consciente de la necesidad de contar con instrumentos cada vez mejores.

Leamos, ahora, lo que nos dice don Jorge Juan acerca del problema de la longitud:

"Varios métodos hay de determinar las longitudes; pero el más exacto (para distancias grandes) que al presente se conoce, es por las observaciones de diferencias en tiempo entre los lugares, cuyas longitudes se pretenden saber: las cuales diferencias también se determinan por varios caminos; siendo el más justo de todos el observar en ambos sitios la misma inmersión o emersión de los satélites de Júpiter: porque viéndose esta por dos observadores al mismo instante, $\mathrm{y}$, siendo notado el tiempo 
en que sucedió, por ambos, la diferencia en tiempo queda concluida, con sólo el cotejo de las dos observaciones; y por consiguiente la diferencia en longitud, reduciendo la de tiempo a partes del Ecuador: todo lo cual es bien sabido de los inteligentes; y esta corta explicación solo sirve para los que no estuviesen tan versados en el asunto."

Juan no se limitó a señalar cuál era el mejor método para determinar la longitud, sino que también enseñó como hacerlo: Recomendaba arreglar con la máxima exactitud un reloj de péndulo y observar con un telescopio de 16 a 20 pies de largo. Era importante registrar el instante en que sucediera la inmersión o emersión de los satélites. Para que la observación fuera exitosa y sus resultados útiles, sugería que hubieran al menos dos observadores. Uno, para que contara los segundos que fueran pasando en el reloj de péndulo y el otro, para que observara con el telescopio la inmersión, estando atento a los segundos que fuera contando su compañero. Juan encarecía que las observaciones se hicieran con el mayor cuidado y precisión posibles, pues un solo minuto de diferencia en el tiempo, produciría un error de cinco leguas en longitud.

En los primeros días de Junio de 1802, Caldas escribió desde Quito anunciando que se preparaba para observar el solsticio de verano. Confiaba que esa observación le proporcionaría información acerca de la amplitud de la eclíptica. ¿De donde habría sacado Caldas estas ideas? La respuesta es nuevamente la misma: de la lectura del libro de Jorge Juan. En la época de la expedición geodésica a Perú, establecer la oblicuidad de la eclíptica era el primer paso necesario para la práctica de la astronomía. Juan sostenía que ese conocimiento era el fundamento de la astronomía, pues una vez establecida, era útil para establecer las ascensiones rectas, las declinaciones del Sol, el curso de los planetas, su verdadero lugar en el cielo, las declinaciones de las estrellas, el gobierno de los relojes; era también necesaria para determinar las longitudes, y otros fenómenos más.

Caldas observó el tránsito de Mercurio por el Sol, ocurrido el 9 de noviembre de 1802, desde Otavalo. Este mismo fenómeno fue observado por los marineros de la Expedición Fidalgo, desde Cartagena y por Humboldt, desde el Callao. Todos ellos observaron la salida del planeta, porque el tránsito había empezado antes de que saliera el Sol. Como el mismo Caldas le escribiera a Mutis, Humboldt le habia escrito desde Trujillo recomendándole que hiciera esta observación. 
A principios del siguiente año, el 21 de Febrero de 1803, observó un eclipse de Sol desde Quito. Como en el caso anterior, este evento astronómico también fue observado desde Cartagena por Juan de Tiscar y Valle, oficial de la Expedición Fidalgo y por Ferrer, desde La Habana. Los resultados de esas observaciones simultáneas desde diferentes lugares, le permitieron a Caldas establecer la longitud de Quito, una de sus mayores metas cientificas.

La llegada del Barón de Humboldt y de Amadeo Bonpland a Santafé en 1800 , trajo a los santafereños noticias acerca de los trabajos hidrográficos que estaban realizando unos marineros al mando de Joaquín Francisco Fidalgo. Humboldt viajó hasta Santafé para conocer a Mutis, quien le informó acerca de Francisco José de Caldas. El cientifico alemán llegó al interior de la Nueva Granada por el Río Magdalena y aprovechó su recorrido para hacer mediciones barométricas, observar la declinación de la aguja, establecer un sinnúmero de latitudes y longitudes, utilizado el cronómetro de M. Berthoud. Acopió suficientes materiales para hacer una carta del Río Magdalena, desde su desembocadura -en Bocas de Ceniza- hasta Honda, que se publicó en Paris en 1816, con algunas modificaciones. Humboldt incorporó la carta de la parte alta del Río Magdalena que Caldas le facilitó, al mapa del curso de ese río en su Atlas, y le dio los créditos correspondientes al científico criollo.

En Enero de 1802, unas semanas después de la llegada del Barón a Ecuador, don Francisco José no cabía de contento. Estaba feliz por la oportunidad de interactuar con un cientifico europeo y orgulloso de todos los elogios que le había hecho el Barón. Consideraba que el trato con el prusiano le había servido para concluir muchos de los trabajos astronómicos que ya había adelantado, sentía haber logrado comprender los "más profundos misterios" de esa ciencia y que ya se podía llamar a sí mismo astrónomo. Del Catálogo de Estrellas, de Flamsteed, que trajo Humboldt, el criollo copió los nombres y posiciones de 440 estrellas, que sumadas a las 120 que ya conocía, lo hacía sentirse dueño de 560 . En cuanto al aprecio que hizo el Barón de sus trabajos, sintió que la aprobación del prusiano coronaba de gloria sus trabajos. Así le escribió a Santiago Arroyo: "Después de leer mis manuscritos dijo en una tertulia que mis observaciones astronómicas están más bien ejecutadas que las de don Jorge Juan. ¿Qué honor para mi oírme preferir a este hombre admirado de la Europa! (...)" 


\section{Director del Observatorio Astronómico de Santafé}

Las tareas geográficas y astronómicas estaban contempladas desde un principio en la solicitud de creación de la Expedición Botánica, que hizo Mutis; el Rey envió desde el comienzo dotaciones de instrumentos astronómicos. Mutis había enseñado el modelo heliocéntrico y la física newtoniana en su Cátedra de Matemáticas del Colegio del Rosario, desde la época en que llegó a Santafé; no era un astrónomo dedicado, pues sus mayores intereses estaban en la Botánica, así que solamente realizó unos cuantos trabajos astronómicos: determinó la latitud de Santafé, observó el eclipse de un satélite de Júpiter que le sirvió para determinar la longitud de Santafé; también hizo observaciones diurnas del barómetro.

En el mismo año de 1801, que mandó a construir el Observatorio Astronómico, Mutis le escribió por primera vez a Francisco José de Caldas anunciándole que lo había agregado a la Expedición, pero el payanés sólo llegó a Santafé en Diciembre de 1805; llegó acompañado de quince mulas cargadas con todo el material recogido durante su estancia en Quito y en su travesía hasta Santafé. Por mandato de Mutis, asumió la dirección del Observatorio Astronómico, desde su llegada. En el número 7 del Semanario, Caldas publicó el artículo "Descripción del Observatorio Astronómico de Santafé de Bogotá, situado en el Jardín de la Real Expedición Botánica". Allí, efectivamente presenta una descripción del edificio y de las tareas que realizó desde que se hizo cargo del establecimiento, incluyendo el montaje de los instrumentos enviados desde España.

Destacó las ventajas del Observatorio Astronómico de Santafé de Bogotá, derivadas de su cercana ubicación al Ecuador, que posibilitaba la observación de las estrellas del Hemisferio Norte y del Hemisferio Sur. Consideraba que todas esas ventajas se aumentarian si dispusiera de mayor cantidad de instrumentos astronómicos, a pesar de que poseía una dotación adecuada de ellos. Se refirió, especialmente, al conocimiento de las estrellas del Sur y a la posibilidad de observar y conocer nuevas estrellas dobles y triples y también de conocer nebulosas. Me importa señalar este detalle; es decir, que Caldas conocía la existencia de estrellas dobles y triples y también, la existencia de nebulosas. Kant y Laplace habian formulado la hipótesis del origen del universo a partir de una nebulosa; pero el concepto mismo de nebulosa no era muy preciso en ese entonces. Todavía no existían medios para saber que todas las nebulosas no eran iguales, que hay nebulosas planetarias, estelares, galácticas, 
cúmulos y otras. Casi contemporáneo con Caldas, Charles Messier (17301817), astrónomo francés interesado en la observación de cometas, con el fin de facilitar la detección de los mismos, hizo un catálogo de las nebulosas presentes en el cielo, que él considera objetos fijos, el famoso Catálogo Messier, publicado en 1781. Alli registró las posiciones exactas de 110 de esos objetos, con el fin de distinguirlos de los buscados cometas. El catálogo, que se inicia con la nebulosa del Cangrejo (MI), contiene otros objetos importantes, como la galaxia de Andrómeda (M31).

Un balance de los trabajos de astronomía realizados desde el Observatorio, se encuentra en el primer Informe al Virrey Amar y Borbón, que envió Caldas el $1^{\circ}$ de Julio de 1809 :

"En los últimos cuatro meses he verificado largos trabajos sobre las refracciones astronómicas al nivel y latitud de este Observatorio. Vuestra Excelencia sabe que este elemento es capital en la Astronomía, y que jamás se trabaja mucho cuando se trabaja con utilidad. Vuestra Excelencia hallará en mi Colección de Observaciones Astronómicas una Memoria sobre las refracciones en Santafé. Todas las alturas meridianas del sol, tan interesantes para las refracciones y tan necesarias para todo cuanto se ejecute en esta ciencia inmensa y sublime, las alturas de las estrellas en las noches que lo han permitido las nubes, el último eclipse de luna, las inmersiones y emersiones de los satélites de Júpiter, las ocultaciones de las estrellas por la Luna, distancias de estos planetas, el último solsticio felizmente observado, las variaciones del barómetro, termómetro e higrómetro tres veces cada día, cálculos dilatados, organización de observaciones y de manuscritos, para adelantar las obras de las que he hablado a su Excelencia, son las ocupaciones que he tenido desde que merecí el encargo de este establecimiento, que hace tanto honor al sabio que lo proyectó, al Reino, a la América y sobre todo, al ilustre Jefe que sabe apreciarlo, y que le sostiene bajo su poderosa protección".

El payanés aprovechó ese informe para solicitarle al Rey que autorizara el envío de las Efemérides astronómicas que publicaba anualmente el Observatorio Real de la Isla de León. Consideraba que, en tanto establecimiento público, el Observatorio debía contar con estas publicaciones, muy abundantes en la península ibérica. También le solicitó al Virrey le permitiera entregar los informes acerca de sus trabajos de Astronomía cada año, y no cada cuatro meses, como estaba obligado. Explicaba que de las otras materias podía dar informes en ese período, pero 
que las observaciones astronómicas requerían al menos un año para que los datos recogidos pudieran adquirir sentido en el contexto de la rotación anual de nuestro planeta.

Los métodos más usados para calcular la longitud en la época de Caldas, requerian un enorme trabajo de cálculos matemáticos después de haber realizado las observaciones, básicamente, de los eclipses de las lunas de Júpiter y de los eclipses de Luna: Era necesario hacer las conversiones de los tiempos de observación de acuerdo al Almanaque Náutico.

Una parte muy importante de la labor que realizó nuestro científico en el Observatorio consistió en la elaboración de efemérides. El $1^{\circ}$ de Enero de 1811 los santafereños leyeron un folleto escrito y publicado por Caldas, que contenía el Almanaque del Nuevo Reino de Granada para 1811, en el cual se detallaban los eclipses de Sol y de Luna de una manera diferente a la acostumbraba en los almanaques de pliego. El Almanaque constaba de las siguientes partes: Prefación, Épocas, (fechas) de la Historia de las Ciencias, Sistema Planetario, Cómputos Eclesiásticos, Cuatro Témporas, Fiestas Móviles, Oblicuidad aparente de la eclíptica, eclipses de Sol y Luna, Apulsos y, para cada uno de los meses del año: Longitud de Sol, declinación del Sol, semidiámetro del Sol, longitud del nodo de la Luna, apogeo y perigeo lunar, puntos notables de la Luna en la órbita y calendario.

Un año después en el Almanaque de las Provincias Unidas del Nuevo Reino de Granada para el año bisiesto de 1812, explicaba que habia puesto al comienzo de cada mes, la longitud, declinación y semidiámetro del Sol, junto con la longitud del nodo ascendente de tarea a las personas que quisieran sumarse a los cálculos de latitud y longitud de los lugares donde habitaban. Fiel a su interés de "difundir las luces", de lograr que cada vez se vinculara mayor cantidad de personas a las tareas científicas de apropiación del medio, insertó, por primera vez, los apulsos que iban a ser visibles en toda la extensión del Reino en ese año y señalaba que ése era uno de los métodos más exactos para la determinación de las longitudes, porque atendía más al rigor geométrico, no exigía aparatos ni mayores gastos; animaba a los lectores a observar estos fenómenos, tarea que consideraba sencilla, inclusive al alcance de personas que no estuvieran instruidas en Astronomía. 
En ese mismo Almanaque de 1812, Caldas expresó su conocimiento acerca de la explicación newtoniana sobre el origen de las mareas. Ofreció definiciones de conceptos astronómicos relacionados con todos los puntos lunares para facilitar la observación aún a personas que no fueran profesionales. Definió zizigia, cuadratura, apogeo, perigeo, lunisticio, equinoccio, Luna eclíptica y cenit.

Don Francisco José tenía un conocimiento bastante actualizado acerca de la historia de las ciencias de su época. Al final presentaba el Sistema Planetario, según los últimos descubrimientos:

Sol, centro del sistema y foco de luz.

Mercurio

Venus

Tierra, con un satélite, o Luna

Marte

Júpiter, con cuatro satélites o lunas

Saturno, con dos anillos concéntricos y cinco lunas.

Herschel, con ocho satélites o lunas

Piazzi, descubierto por Mr. Piazzi, astrónomo de Palermo

Olbers, descubierto por M. Olbers

Harding, descubierto por Mr. Harding

Hay, pues, en nuestro sistema doce planetas primarios, dos anillos y diez y ocho secundarios; entre todos, treinta y dos cuerpos opacos que ruedan alrededor del Sol.

El Sol, Mercurio, Venus, la Tierra, la Luna, Marte, Júpiter y Saturno eran las siete estrellas errantes conocidas desde la antigüedad. Herschel, Piazzi, Olbers, Harding fueron nombres provisionales correspondientes a los descubridores de algunos cuerpos celestes.

El 1 de enero de 1801, Giuseppe Piazzi descubrió un cuerpo celeste. En un primer momento supuso que se trataba de un nuevo cometa; sin embargo, después de determinar su órbita, quedó claro que se parecía más a la de un planeta. Piazzi lo bautizó Ceres, en honor a la patrona siciliana de la agricultura. Se trataba de un asteroide, el mayor de los que forman el cinturón de asteroides.

William Herschel descubrió el planeta Urano, después de analizar las perturbaciones en la órbita de Saturno, calculó en qué órbita podría en- 
contrarse el cuerpo celeste que las provocaba; enfocó su telescopio a ese lugar del cielo y efectivamente lo encontró! El descubrimiento de Urano constituyó una confirmación a la Mecánica de Newton. Así que el planeta Herschel es Urano.

¿Qué cuerpo celeste descubrió Olbers, para que mereciera llevar su nombre, al menos por un tiempo? H. W. Matthäus Olbers (1758-1840), fue médico de profesión y astrónomo aficionado. En 1779 creó el primer método, todavía utilizado hoy en día por los astrónomos, para calcular la órbita de los cometas. El 1 de enero de 1802 Olbers localizó, en la posición prevista por Karl F. Gauss, el primer asteroide, Ceres, que ya habia sido descubierto un año antes por Giussepe Piazzi, y después se habia perdido de vista. Poco tiempo después, siguiendo a Ceres, Olbers descubrió otro asteroide, Palas, y se convenció de que estaban relacionados con los fragmentos de un cuerpo más grande; por lo tanto buscó otros fragmentos y en 1807 descubrió Vesta. Descubrió cinco cometas y calculó la órbita de 18.

Caldas comprendía bien cómo se podía usar la observación del comportamiento del Sol, la Luna y las estrellas para comprender la ubicación geográfica. En su artículo "La Expedición Botánica y la Comisión Corográfica. Una mirada comparativa" Olga Restrepo sostiene que la Geografia fue el hilo conductor de los trabajos del científico neogranadino que venimos estudiando:

La astronomía, las matemáticas o la botánica, como disciplinas independientes, no permiten dar coherencia y sentido a la obra de Caldas; no representaban el hilo conductor de sus intereses y su actividad de indagación. Ese hilo conductor no era otro que la geografia. La inclinación de Caldas por la geografia le exigió ampliar sus conocimientos de astronomia; estudio botánica desde el horizonte de la distribución espacial de las plantas.

Por su parte, en la Memoria sobre el plan de un viaje proyectado de Quito a la América Septentrional, presentada al célebre Director de la Expedición Botánica de la Nueva Granada, Don José Celestino Mutis, por F.J. de Caldas, escrito probablemente en 1801, Caldas dio a conocer su concepción de Astronomía, al señalar las tareas relativas a esta materia de las que se ocuparía si su proyecto fuese aprobado:

Las longitudes y latitudes de los lugares, y sobre todo de Guayaquil, Sonsonate, Veracruz, etc., serán nuestro primer cuidado. Usaremos de 
todos los métodos conocidos: eclipses de sol, de luna, satélites de Júpiter, distancias de la luna al sol, paso por el meridiano de aquella, etc. En nuestras travesias por mar comprobaremos por nuestras propias observaciones la exactitud de los métodos.

Para su trabajo cartográfico, construido a partir de observaciones astronómicas, Francisco José utilizó la Astronomía de Lalande, la versión anual del Almanaque Náutico de Cádiz, la Guía de Navegantes, las Observaciones Astronómicas de Jorge Juan y el Atlas Celeste de Flamsteed que le permitió consultar Humboldt cuando se encontraron en 1802. Desde 1797 había emprendido el estudio de los mapas más conocidos, incluyendo los de Piedrahita y Maldonado sobre la Nueva Granada; además le encargó a Santiago Arroyo que le enviara a Popayán el mapa de América Meridional de Juan de la Cruz Cano y Olmedilla. De Geografia, Caldas conoció los trabajos de algunos jesuitas: Marañón, Mayn, Gumilla, a quienes tomó como referencia.

La mayor parte de los trabajos cartográficos de Francisco José de Caldas corresponden a la época en que fue mercader y recorrió los territorios de la Nueva Granada desde Popayán hasta el sur de Ecuador, es decir, entre 1797 y 1805 .

A fines de 1798 el acopio de datos que había ido recogiendo en sus correrías, lo llevaron a acariciar el proyecto de elaborar el mapa de todo el virreinato. Ya disponía regularmente del Almanaque Náutico de Cádiz y de un telescopio, podía usar los fenómenos de los satélites de Júpiter que observaba, para agregar a sus mapas más longitudes fijadas astronómicamente. Una de las observaciones de este fenómeno es recordada en nuestra tradición histórica, la de la emersión del primer satélite de Júpiter ocurrida el 22 de diciembre de 1798, con la que fijó la longitud de Popayán; Humboldt mismo admiró este trabajo, cuyos datos encontró muy acordes con los suyos propios. Al mismo tiempo le sirvió como referencia para las demás determinaciones de longitud que realizó en su correría por la América Meridional.

Su primer trabajo cartográfico fue la carta de la Provincia de Timaná que hizo a instancias de los gobernantes de la misma que requerían dirimir un pleito de límites con el cabildo de La Plata. Para realizarla, tuvo que determinar numerosas latitudes, realizar operaciones de triangulaciones y lograr la longitud de algún lugar. Un fenómeno astronómico vino a ayudarlo en esta última tarea, el eclipse total de Luna del 3 al 4 de 
diciembre de 1797, que observó en Gigante. Así pues, su primera determinación astronómica de longitud fue un excelente trabajo.

El encuentro con Humboldt y Bonpland, le confirmó a Caldas la importancia de la geografia. Se propuso llevar a cabo una Geografia Botánica de las plantas del Virreinato, que estudiaría las alturas a las que se producian las plantas en este territorio, tal como escribió en Estado de la Geografia del Virreinato de Santafé..., en 1807, donde propuso la integración de una expedición geográfica o económica e itinerante.

Entre septiembre y diciembre de 1802 viajó al norte de Quito, Ibarra y Otavalo, donde observó el paso de Mercurio frente al disco solar, del que ya hablamos. Entre julio y agosto de 1803 hizo el reconocimiento y exploración del camino de Malbucho, buscando una salida al mar a instancias de Carondelet; este trabajó le permitió determinar astronómicamente Ibarra, Salinas, Malbucho, San Miguel y otros. En mayo de 1804 viajó a Barnuevo, permaneció en Quito para el solsticio de junioy entre julio y diciembre viajó a Riobamba, Cuenca y Loja. Entre otros lugares determinados astronómicamente están Quito, Turabamba, Machache, Sasquisili, Tagualó, Otavalo, Ibarra, Guaca, Guachacal, Santa Lucía, Popayán, Matarredonda, Macuchí, Ambato, Riobamba y Cuenca. Además levantó la carta de casi toda la parte meridional de la Nueva Granada. Cuando se reunió con Humboldt le entregó un mapa del alto Magdalena, con las respectivas observaciones astronómicas y cálculos sobre la posición de Popayán y otros puntos intermedios hasta Ibarra. El Barón publicó este mapa en su Atlas Geográfico de 1814.

En su Informe al Virrey (1809), del que ya hablamos, don Francisco José reconocía los aportes que habían hecho a la cartografia Pedro Maldonado, La Condamine, Fidalgo y Humboldt, pero se enorgullecia de haber sido quien había aclarado muchas dudas y quitado muchos errores de los trabajos cartográficos realizados previamente. Consideraba que los conocimientos geográficos eran el parámetro con que se median la ilustración, el comercio, la agricultura y la prosperidad de un pueblo. También era consciente de la importancia de la geografia para los gobernantes, porque ella les daba a conocer las dimensiones del territorio que gobernaban, la riqueza de las costas, cuáles eran los ríos navegables, dónde estaban las montañas y los valles, los lugares donde habian caminos y donde se podrían trazar nuevos caminos, el clima, la temperatura, la elevación sobre el mar de todos los puntos, las costumbres de sus 
habitantes, las producciones espontáneas y las que podrian "connaturalizarse" con el arte. Inclusive propuso métodos para abrir nuevos caminos, que atravesaran las cordilleras.

Para finalizar, nos referiremos brevemente al proyecto que presentó Caldas desde el Semanario para que se formara una expedición geográfica o económica destinada a recorrer el Virreinato, que debía estar compuesta de un astrónomo, de un botánico, de un mineralogista, de un encargado de la parte zoológica y de un economista, a los que se unirian dos dibujantes y podría ser financiada por todas las Provincias, que contribuirían con un fondo formado por las personas más adineradas, por los comerciantes y por el Consulado de Cartagena. La expedición no se realizó, pero su propuesta de conocer mejor nuestro territorio encontró eco en algunos neogranadinos, que enviaron sus contribuciones al Semanario. 


\section{Bibliografía}

ARIAS DE GREIFF, Jorge y otros, CALDAS 1768-1816. Francisco Joseph de Caldas y Thenorio. Edición conmemorativa a los veinticinco años del Instituto Colombiano para el Desarrollo de la Ciencia y la Tecnología Francisco José de Caldas "Colciencias". Bogotá, Molinos Velásquez Editores, 1994.

ARIAS DE GREIFF, Jorge, La astronomía en Colombia, Bogotá, Academia Colombiana de Ciencias Exactas, Físicas y Naturales, $1^{\text {a }}$ ed., 1993.

BATEMAN, Alfredo, El Observatorio Astronómico de Bogota. Monografia histórica con ocasión del $150^{\circ}$ aniversario de su fundación. Bogotá, Universidad Nacional 1953.

CALDAS Y TENORIO, Francisco José, Cartas de Caldas, Bogotá, Academia Colombiana de Ciencias Exactas, Físicas y Naturales, 1978.

CALDAS Y TENORIO, Francisco José, Obras Completas de Francisco José de Caldas, Bogotá, Imprenta Nacional, 1966.

CALDAS Y TENORIO, Francisco José, Semanario del Nuevo Reino de Granada, Bogotá, Biblioteca Popular de Cultura Colombiana, Editorial Kelly, 1942.

COLCIENCIAS, Historia Social de la Ciencia en Colombia. Tomo II, Matemáticas, Astronomía y Geología. Bogotá, Tercer Mundo, 1993.

DIAZ PIEDRAHITA, Santiago, Nueva aproximación a Francisco José de Caldas. Episodios de su vida y de su actividad cientifica. Bogotá, Academia Colombiana de Historia, 1997.

FONNEGRA, Gabriel, Compilador, Mutis y la Expedición Botánica. Documentos. Bogotá, El Ancora Editores, 1983.

GONZALEZ PEREZ, Marco, Francisco José de Caldas y la Ilustración en la Nueva Granada, Bogotá, Ediciones Tercer Mundo, 1984.

GREDILLA Y GAUNA, Apolinar Federico, Biografia de José Celestino Mutis; con la relación de su viaje y estudios particulares en el Nuevo Reino de Granada. Madrid, Establecimiento Tipográfico de Fortanet, 1911.

HERNANDEZ DE ALBA, Gonzalo, Pensamiento científico y filosófico de José Celestino Mutis, Bogotá, Fondo Cultural Cafetero, 1982.

HERNANDEZ DE ALBA, Guillermo, Compilador, Archivo Epistolar del Sabio Naturalista Don José Celestino Mutis, Bogotá, Editorial Kelly, 19681975.

HERNANDEZ DE ALBA, Guillermo, Compilador, Escritos científicos de Don José Celestino Mutis, Bogotá, Editorial Kelly, Instituto Colombiano de Cultura Hispánica, 1983.

JUAN, Jorge, Observaciones astronómicas y phisicas hechas de orden de S. Mag. en los Reinos del Perú, por Jorge Juan, Comendador de Aliaga en el Orden de S. Juan, Socio Correspondiente de la R. Academia de las Ciencias de París, y D. ANTONIO DE ULLOA, de la R. Sociedad de Londres, ambos Capitanes de Fragata de la $\mathrm{R}$. Armada, de las quales se deduce la figura 
y magnitud de la Tierra y se aplica a la Navegación. Impreso de orden del Rey Nuestro Señor en Madrid por Juan Zúñiga, Año MDCCXLVIII.

LAFUENTE, Antonio y MAZUECOS, Antonio, Los caballeros del punto fijo.

Ciencia y aventura en la expedición geodésica al Perú. Quito, ABYA-YALA, 1992.

LALANDE, M. de, Connoissance des mouvemens celestes pour l'année comune 1765. Publié par l'ordre de l'Academie Royale des Sciences. Paris, MDCCLXIII.

MASKELYNE, Nevil, The Brithish Mariner's Guide. Containing complete and easy instructions for the discovery of the longitude at sea and land, within a degree, by observations of the distance of the Moon from the Sun and stars, by Nevil Maskelyne A. M. Fellow of Trinity College, Cambridge, and of the Royal Society, London, in-4, 1763.

NEWTON, Isaac, Principios Matemáticos de la Filosofia Natural y su Sistema del Mundo. Edición preparada por Antonio Escohotado. Madrid, Editora Nacional, 1982.

OLIVOS LOMBANA, Andrés, Caldas. Precursor del patriotismo cientifico. Bogotá, Panamericana Editorial, 1998.

RESTREPO, José Félix, Obras completas. Bogotá, Biblioteca Colombiana de Filosofia, Universidad Santo Tomás, 2002.

RESTREPO, Olga, "La Expedición Botánica y la Comisión Corográfica: Una mirada comparativa" en Senderos. Publicación Semestral de Biblioteca Nacional de Colombia (Bogotá), 5(25/26): 535-563. Primer Semestre, Agosto de 1993.

SELLES, Manuel A., Astronomía y navegación en el siglo XVIII. Madrid, Ediciones AKAL, 1992.

SELLES, PESET y LAFUENTE, Carlos III y la ciencia de la Ilustración. Madrid, Alianza Universidad, 1987.

SOBEL, Dava, Longitud. Madrid, Editorial Debate, $1^{\text {a }}$ ed., 1998.

VEZGA, Florentino, La Expedición Botánica. Cali, Carvajal \& Cía, 1971

\section{Recibido: mayo 2007}

Aprobado: septiembre 2007 